\title{
Containerless processing by single-axis electrostatic levitation
}

\author{
HU Liang, WANG HaiPeng, XIE WenJun \& WEI BingBo \\ Department of Applied Physics, Northwestern Polytechnical University, Xi'an 710072, China
}

Containerless state is one of the main characteristics of the space environment, which avoids the contamination from crucibles, and provides a special condition to materials research in outer space. Various levitation techniques have been established to realize the containerless state on the ground. Electrostatic levitation applies the Coulomb force on the charged sample in an electrostatic field to counteract the gravity and achieve containerless state. Compared with other levitation techniques, electrostatic levitation has many advantages: It is applicable to various kinds of materials that holds sufficient surface electric charges, such as metals, oxides and polymers. Its levitation stability can be maintained in a very broad temperature range, since the levitation and heating are controlled independently. It exerts negligible disturbance to the inner part of the sample, and hence is favorable for the achievement of high undercooling and rapid solidification of liquid alloys. In addition, it can be conducted in vacuum and eliminates the influence of gas medium. Therefore, electrostatic levitation becomes an important approach to the study of containerless processing for advanced materials.

Since there exists no three-dimensional potential well in the electrostatic field, great difficulties are brought about to the research on the control of levitation stability. In the recent decades, containerless processing of materials by electrostatic levitation were performed in NASA (National Aeronautics and Space Administration, USA), DLR (German Aerospace Center) and JAXA (Japan Aerospace Exploration Agency). Supported by the Creative Research Team Project of the National Natural Science Foundation of China, an electrostatic levitator with single-axis feedback control system was developed by Professor Wei Bingbo's group in Northwestern Polytechnical University. Both experimental and theoretical investigations were accomplished to solve the four key problems of electric field optimization, sample position detecting, sample charging control and levitation voltage minimization for stable levitation. Various types of materials, such as metals (AZ91D magnesium alloy and ball of aluminum foil), inorganic materials (graphite, $\mathrm{C} / \mathrm{C}$ composite materials, red phosphorus and silicon dioxide) and organic materials (carbon fiber reinforced resin, liquid crystal and mung bean) have been successfully levitated in the experiments. The typical levitation experiment results are illustrated in Figure 1. The levitated sample exhibits fast control response and satisfactory levitation stabilization. It takes only $0.1 \mathrm{~s}$ to realize the stable levitation in upright direction, and the fluctuation is smaller than $0.3 \mathrm{~mm}$ (Figure 2(a)). The constraining force produced by spherical electrodes ensures the horizontal stability with a small fluctuation below $0.5 \mathrm{~mm}$ (Figure 2(b)).
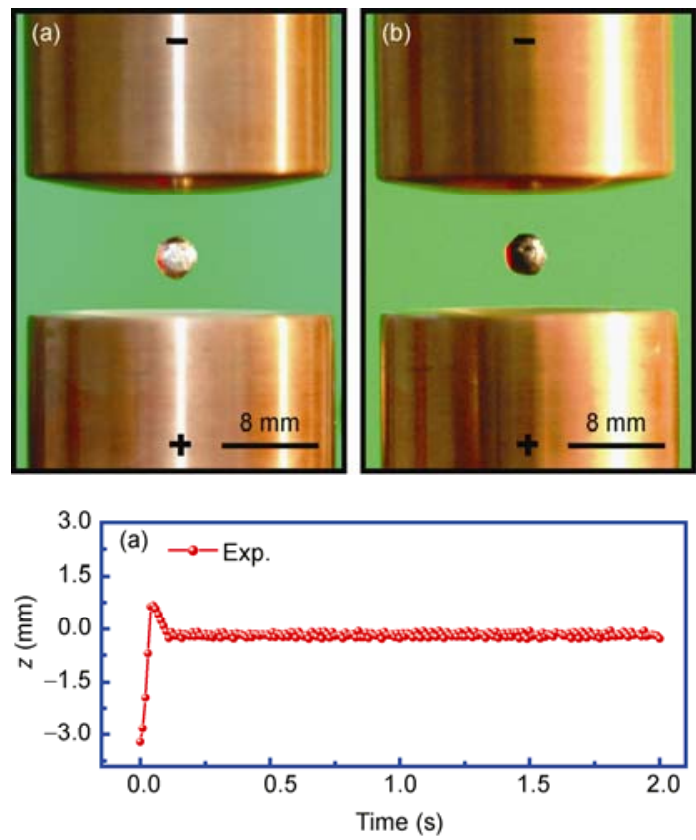
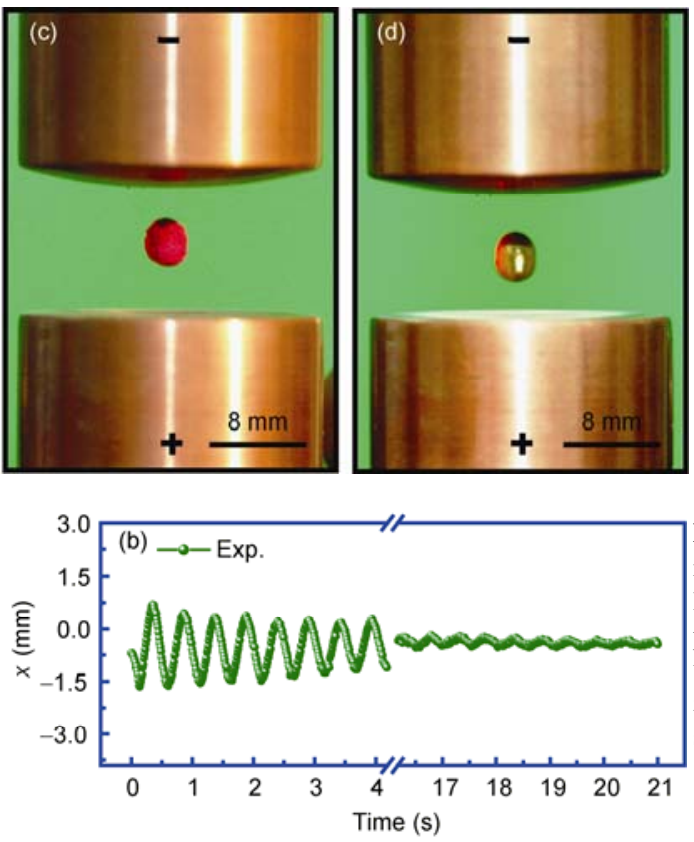

Figure 1 Electrostatic levitation of typical materials. (a) Magnesium alloy; (b) graphite; (c) red phosphorus; (d) mung bean.

See the article: Hu L, Wang H P, Xie W J, et al. Electrostatic levitation under single-axis feedback control condition. Sci China Phys Mech Astron, 2010, 53: 1438-1444 